\title{
Nuclear Maps and Modular Structures II: Applications to Quantum Field Theory
}

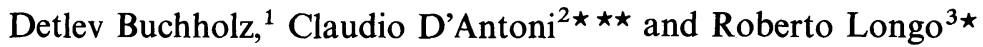 \\ ${ }^{1}$ II. Institut für Theoretische Physik, Universität Hamburg, D-2000 Hamburg 50, Federal Republic of \\ Germany \\ 2 Dipartimento di Matematica, Università di Roma "La Sapienza", P.le A. Moro 5, I-00185 Roma, Italy \\ ${ }^{3}$ Dipartimento di Matematica, Università di Roma "Tor Vergata", Via Fontanile de Carcaricola, \\ I-00133 Roma, Italy
}

\begin{abstract}
A correspondence between spectral properties of modular operators appearing in quantum field theory and the Hamiltonian is established. It allows to prove the "distal" split property for a wide class of models. Conversely, any model having this property is shown to satisfy the Haag-Swieca compactness criterion. The results lead to a new type of nuclearity condition which can be applied to quantum field theories on arbitrary space-time manifolds.
\end{abstract}

\section{Introduction}

The physical significance of the modular operators appearing in quantum field theory is still a mystery: given the von Neumann algebra $\mathscr{A}(\mathcal{O})$ of operators which are associated with a space-time region $\mathcal{O}$ and given the vector $\Omega$ representing the vacuum state, the Tomita-Takesaki theory [3] provides us on the one hand with a one-parameter group of unitaries $\Delta^{i t}, t \in \mathbb{R}$ (the modular group) which induces automorphisms of $\mathscr{A}(\mathcal{O})$. Moreover, the restriction of the vacuum state to $\mathscr{A}(\mathcal{O})$ behaves like an equilibrium state under the action of these automorphisms (it satisfies the KMS-condition). These mathematical facts seem to suggest that the modular group is some kind of dynamics of an observer in the region $\mathcal{O}$.

On the other hand, all attempts to justify such a physical interpretation in general have failed so far. There is only a single, though important special case where this interpretation is known to be correct: if $\mathcal{O}$ is a wedge-shaped region which is bounded by two characteristic planes, it can be shown that the corresponding modular group is the dynamics of a uniformly accelerated observer [21]. But for arbitrary regions such a clearcut interpretation is out of sight.

As a step towards a clarification of this point we exhibit in the present investigation a tight relation between spectral properties of the modular operators and the Hamiltonian $H$. Although our results do not solve the problem of the

* Supported by the A. von Humboldt Stiftung, Bonn

$\star \star$ Supported in part by Ministero della Pubblica Instruzione and CNR-GNAFA 
interpretation of the modular operators, they are perfectly consistent with (and in fact were suggested by) the idea that these groups are perturbed dynamics.

Our starting point is the observation made in $[17,9]$ that in theories with a physically reasonable number of local degrees of freedom the set of vectors

$$
e^{-\beta H} \mathscr{A}_{1}(\mathcal{O}) \Omega, \quad \beta>0,
$$

where $\mathscr{A}_{1}(\mathcal{O})$ denotes the unit ball of $\mathscr{A}(\mathcal{O})$, should be "small" (i.e. compact or nuclear) if the region $\mathcal{O}$ is bounded. A heuristic argument leading to this conclusion is the following one (cf. [9] and Sect. 5): the operator $e^{-\beta H}$ in (1.1) is restricted to excitations of the vacuum in a bounded region, properties at spacelike infinity are not tested. Disregarding boundary effects the size of the set (1.1) should therefore not change very much if one proceeds from the original theory to the corresponding theory in a sufficiently large but finite volume $V$ (a "box"). In this passage the operator $e^{-\beta H}$, which has continuous spectrum, is replaced by the operator $e^{-\beta H_{V}}$, which in the physical setting is to be interpreted as the density matrix of the Gibbs-ensemble in the volume $V$ at temperature $\beta^{-1}$. It therefore ought to be of trace class. The smallness of the set of vectors in (1.1) in the sense indicated is then an immediate consequence.

We note that if one proceeds from (1.1) to the corresponding expression in a representation of the local algebras which is induced by an equilibrium state at temperature $T$, one arrives at a similar result (cf. Sect. 5): if $\Omega$ now stands for the cyclic vector representing this equilibrium state and $H$ for the generator of the time translations (which is no longer bounded below in this case), then the set of vectors (1.1) should still be small, provided $\beta$ is restricted to $0<\beta<1 / 2 T$.

Taking the idea seriously that the modular group is some kind of a dynamics, one would expect that an observer in the region $\mathcal{O}$, whose time evolution is given by the modular group, comes to a similar conclusion about the size of the set of vectors which, according to his view, corresponds to (1.1). In order to find the appropriate counterpart of this set two remarks are in order: first, the special example of the uniformly accelerated observer suggests that the boundaries of the underlying region $\mathcal{O}$ should be regarded as horizons, playing the same rôle as infinity for an observer in Minkowski space. Second, the vacuum state is not a ground state for the generator $K=-\ln \Delta$ of the modular group. But, as already mentioned, it can be interpreted as an equilibrium state whose temperature is normalized to one. Taking these remarks into account we were led to consider as analogue of (1.1) the set of vectors

$$
e^{-\lambda K} \cdot \mathscr{A}_{1}\left(\mathcal{O}_{0}\right) \Omega, \quad 0<\lambda<1 / 2,
$$

where $\mathcal{O}_{0}$ is any bounded region whose closure is contained in the interior of $\mathcal{O}$. The restriction of $\mathcal{O}_{0}$ arises from the condition that $e^{-\lambda K}$ should only locally be tested, but not at the horizon, and the limitations on $\lambda$ conform with the previous comment on the properties of the set (1.1) in the case of equilibrium states.

The main result of the present investigation is the observation that the sets (1.1) and (1.2) have indeed comparable sizes. Namely there exists a tight relation between their respective $\varepsilon$-contents. For a brief explanation of this notion and some related material cf. Sect. 2. The precise quantitative information about the relation between the sets (1.1) and (1.2) is given in Sect. 3. 
Besides the more speculative aspects connected with the interpretation of the modular groups there are several concrete consequences of our results which we want to indicate now. The first application relates to the question of whether a quantum field theory has the split property, i.e. whether for given regions $\mathcal{O}_{1} \subset \mathcal{O}_{2}$ there exists a factor $\mathscr{M}$ of type $I$ such that

$$
\mathscr{A}\left(\mathcal{O}_{1}\right)^{\prime \prime} \subset \mathscr{M} \subset \mathscr{A}\left(\mathcal{O}_{2}\right)^{\prime \prime} \text {. }
$$

If $\mathcal{O}_{1}$ is bounded and $\mathcal{O}_{2}$ is any region containing the closure of $\mathcal{O}_{1}$ in its interior, then this question is known to have an affirmative answer whenever the sets (1.1) satisfy the condition of nuclearity proposed in $[9,5]$. Theories with a maximal (Hagedorn-) temperature violate this condition, but the present results allow to establish the existence of intermediate type $I$ factors also in these cases, provided the distance between the boundaries of the regions $\mathcal{O}_{1}$ and $\mathcal{O}_{2}$ in (1.3) is sufficiently large. Simple examples of such models have been studied in [8] and [11]. The present results show that the connection between the rapid growth of the energy-level density in these models and the existence of a maximal temperature and minimal splitting distance is in fact a quite general phenomenon.

A partial converse of these results is the observation that the sets (1.1) must be compact in all theories having the (distal) split property. This is of interest since it provides evidence to the effect that relevant information about the energymomentum spectrum of a quantum field theory is encoded in gross properties of the underlying net of local algebras. A better understanding of this point is a prerequisite for the general solution of problems relating to the particle interpretation of a quantum field theory [4]. As another application we find that interacting theories in 2 dimensions, such as the $\phi^{4}$-theory and the Yukawa-theory satisfy the Haag-Swieca compactness criterion [17]. These results which are related to the split property will be discussed in Sect. 4.

Finally, we reconsider in Sect. 5 the nuclearity condition proposed in [9] in the light of the present results. We propose an alternative formulation of this condition which is based on the notion of $\varepsilon$-content. This formulation has the advantage that, on the one hand, it contains more detailed information about the sets (1.1), on the other hand it carries directly over to the sets (1.2) involving the modular operators. In the latter case our nuclearity condition is strictly local in the sense that it only requires information about the restriction of the vacuum state to the local algebras. In fact the vacuum does not play any special rôle and can be replaced by a dense set of vectors. It is therefore possible to extend our nuclearity condition to quantum field theories on arbitrary space-time manifolds.

We will supply some heuristic arguments, based on a discussion in [9], which make plausible that our condition ought to be satisfied in theories of physical interest. For the simple example of a free field theory we will establish this fact also by explicit calculations which are relegated to the Appendix.

The setting used in the present investigation is the familiar framework of algebraic quantum field theory [15] amended by some information about the modular operators for wedge-shaped regions, which has been obtained by Bisognano and Wichmann using Wightman fields [2]. In order to establish our notation we briefly list the relevant assumptions.

We consider a net of $C^{*}$-algebras $\mathscr{A}(\mathcal{O})$ assigned to the space-time regions $\mathcal{O}$ 
and acting on a Hilbert space $\mathscr{H}$. We assume that isotony holds, i.e.

$$
\mathscr{A}\left(\mathcal{O}_{1}\right) \subset \mathscr{A}\left(\mathcal{O}_{2}\right) \text { if } \mathcal{O}_{1} \subset \mathcal{O}_{2},
$$

but we will make no explicit use of locality. The Lorentz-transformations $\Lambda$ and space-time translations $x$ are represented by automorphisms $\alpha_{\Lambda, x}$ of the net which act according to

$$
\alpha_{\Lambda, x}(\mathscr{A}(\mathcal{O}))=\mathscr{A}(\Lambda \mathcal{O}+x)
$$

and which are unitarily implemented on $\mathscr{H}$. The generator $H$ of the time translations with respect to a fixed Lorentz-frame (the Hamiltonian) is assumed to be non-negative and to have the simple eigenvalue 0 with corresponding normalized eigenvector $\Omega$ (vacuum). This vector is assumed to be cyclic for $\mathscr{A}(\mathcal{O})$ and $\mathscr{A}(\mathcal{O})^{\prime}$ (the commutant of $\mathscr{A}(\mathcal{O})$ in $\mathscr{B}(\mathscr{H})$ ) whenever the region $\mathcal{O}$ has a spacelike complement with open interior. The specific information about the modular operators for the algebras $\mathscr{A}(W)$ corresponding to wedge-shaped regions $W$ and $\Omega$ will be recalled at the place where it is needed (Sect. 3).

We conclude this introduction with the remark that several of our results (in particular in Sect. 4) illustrate the general findings in [6]. But the present paper is self-contained and does not rely on details of that study.

\section{Nuclear Maps}

We will make use of various concepts and results from the theory of nuclear mappings, as described for example in the monograph of A. Pietsch [20]. For the convenience of the reader we recall here some relevant facts. Most of the subsequent material is well known, but some of the results are only implicitly contained in the literature.

Let $\mathscr{E}$ be any (real or complex) normed vector space with norm $\|\cdot\|$. The unit ball of $\mathscr{E}$ will be denoted by $\mathscr{E}_{1}$, and the (topological) dual of $\mathscr{E}$ by $\mathscr{E}^{*}$. We will discuss various properties of continuous linear mappings $\Theta$ from $\mathscr{E}$ into some normed space $\mathscr{F}$. The norm of these mappings is given by

$$
\|\Theta\|=\sup \left\{\|\Theta(E)\|: E \in \mathscr{E}_{1}\right\} .
$$

We adopt the following terminology from [20].

Definition. A continuous linear mapping $\Theta: \mathscr{E} \rightarrow \mathscr{F}$ is said to be

i) compact if the image of $\mathscr{E}_{1}$ under the action of $\Theta$ is a precompact subset of $\mathscr{F}$.

ii) type $l^{p}, p>0$ if there exists a sequence of linear mappings $\Theta_{l}: \mathscr{E} \rightarrow \mathscr{F}$ of rank $l$ such that

$$
\sum_{i=0}^{\infty}\left\|\Theta-\Theta_{\imath}\right\|^{p}<\infty
$$

iii) type $s$ if it is of type $l^{p}$ for all $p>0$.

We note that each mapping $\Theta$ of type $l^{p}, 0<p \leqq 1$, is nuclear [20; Proposition 8.4.2]. In fact, there exist sequences of linear functionals $e_{\iota} \in \mathscr{E}^{*}$ and of elements 
$F_{1} \in \mathscr{F}$ such that (in the sense of absolute convergence)

$$
\Theta(E)=\sum_{\imath=1}^{\infty} e_{\imath}(E) \cdot F_{\imath} \text { for all } E \in \mathscr{E}
$$

and

$$
\sum_{i=1}^{\infty}\left\|e_{\imath}\right\|^{p \cdot} \cdot\left\|F_{\imath}\right\|^{p}<\infty
$$

One can therefore introduce a quasi-norm on these maps (called $p$-norm), setting

$$
\|\Theta\|_{p}=\inf \left(\sum_{l=1}^{\infty}\left\|e_{l}\right\|^{p \cdot}\left\|F_{\imath}\right\|^{p}\right)^{1 / p}
$$

where the infimum is to be taken with respect to all possible representations of $\Theta$ of the form (2.2). There is also a certain converse of these statements: if a map $\Theta$ has finite $p$-norm for some $0<p<1$, then $\Theta \in l^{p /(1-p)}$. In particular $\Theta$ is of type $s$ if $\|\Theta\|_{p}<\infty$ for all $p>0$ [20; Theorem 8.5.6]. It is also noteworthy that all mappings of type $l^{p}, p>0$ are compact [20; Proposition 8.2.6].

We will frequently make use of the fact that the type of a given mapping can be determined if one has sufficient information on the size of its range. As a convenient measure for this size we introduce (by a slight abuse of the terminology in [20]) the notion of $\varepsilon$-content of a mapping.

Definition. Let $\Theta: \mathscr{E} \rightarrow \mathscr{F}$ be a continuous linear mapping and let, for given $\varepsilon>0$, $N(\varepsilon)$ be the maximal number of elements $E_{t} \in \mathscr{E}_{1}, \quad l=1, \ldots, N(\varepsilon)$ such that $\left\|\Theta\left(E_{l}-E_{k}\right)\right\|>\varepsilon$ if $l \neq k$. (Note that $N(\varepsilon)$ is finite if $\Theta$ is compact.) The number $N(\varepsilon)$ is called the $\varepsilon$-content of $\Theta$.

It is clear that the $\varepsilon$-content $N(\varepsilon)$ of a mapping increases if $\varepsilon$ decreases, and $N(\varepsilon)$ tends to $\infty$ (unless $\Theta$ is the zero-map), if $\varepsilon$ approaches 0 .

Definition. Let $\Theta: \mathscr{E} \rightarrow \mathscr{F}$ be a continuous linear mapping. The non-negative number (if it exists)

$$
q=\limsup _{\varepsilon>0} \frac{\ln \ln N(\varepsilon)}{\ln 1 / \varepsilon}
$$

is called the order of $\Theta$. In order to simplify terminology we frequently say (somewhat sloppily) that a map is of order $q$ if its order is not larger than $q$.

In the subsequent lemma we establish some useful connections between the type and the order of a mapping.

Lemma 2.1. Let $\Theta: \mathscr{E} \rightarrow \mathscr{F}$ be a continuous linear mapping. Then:

i) $\Theta$ is compact iff its $\varepsilon$-content $N(\varepsilon)$ is finite for every $\varepsilon>0$.

ii) $\Theta$ is of type $l^{p}, p>0$ if its order is smaller than $p /(2 p+1)$. Conversely, any mapping $\Theta$ of type $l^{p}, p>0$ is at most of order $p$.

iii) $\Theta$ is of type s iff it is of order 0 .

Proof. The first part of this statement is trivial, and the third part is an immediate consequence of the second part. For the proof of the second assertion we heavily 
rely on the basic results expounded in [20]. Combining Lemma 2 in Sect. 9.6.3 of [20] with Lemma 9.1.6 it follows that for every compact mapping $\Theta$ there exists a family of mappings $\Theta_{\imath}$ of rank $\imath$ such that the inequality

$$
\prod_{\imath=0}^{n}\left\|\Theta-\Theta_{\iota}\right\| \leqq 2[(n+1) !]^{2} \varepsilon^{n+1} N(\varepsilon)
$$

is valid for all $n \in N$ and $\varepsilon>0$. Since one may always choose mappings $\Theta_{l}$ such that $\left\|\Theta-\Theta_{\imath+1}\right\| \leqq\left\|\Theta-\Theta_{l}\right\|$ we see from the above estimate and Stirling's formula that

$$
\left\|\Theta-\Theta_{n}\right\| \leqq(n+1)^{2} \varepsilon N(\varepsilon)^{1 /(n+1)} .
$$

Setting $\varepsilon=(n+1)^{-1 / q^{\prime}}$, where $q^{\prime}$ is any real number which is larger than the order $q$ of $\Theta$, we arrive at

$$
\left\|\Theta-\Theta_{n}\right\| \leqq \text { const } \cdot(n+1)^{2-1 / q^{\prime}} .
$$

This shows that $\Theta \in l^{p}, p>0$ if $q<p /(2 p+1)$. Conversely, if $\Theta \in l^{p}$, i.e. if there exists a sequence of mappings $\Theta_{l}$ of rank $l$ such that

$$
\sum_{i=0}^{\infty}\left\|\Theta-\Theta_{\imath}\right\|^{p}=c<\infty,
$$

there holds for any $n \in N$ the estimate

$$
(n+1) \cdot\left\|\Theta-\Theta_{n}\right\|^{p} \leqq c .
$$

Hence $\left\|\Theta-\Theta_{n}\right\| \leqq \varepsilon / 3$ if $n+1 \geqq c(3 / \varepsilon)^{p}$ and it then follows by combining Lemma 9.1.6 with Lemma 1 in Sect. 9.6.3 of [20] that

$$
N(\varepsilon) \leqq(3\|\Theta\| / \varepsilon+2)^{c(3 / \varepsilon) p} .
$$

The statement about the order of $\Theta$ is an immediate consequence of this estimate.

The relation between the order and the type of a mapping given in the second part of this lemma leaves a certain margin which can be narrowed down with some effort in the case of the mappings considered below. Since these improved bounds have little effect on our final results we refrain from giving details.

For later reference we need also

Lemma 2.2. The continuous linear mappings $\Theta: \mathscr{E} \rightarrow \mathscr{F}$ of fixed type $l^{p}, p>0$ (respectively of fixed order $q \geqq 0$ ) form a linear space.

Proof. The first half of this statement has been taken from [20, Proposition 8.2.2]. For the proof of the second half we note that if $\Theta_{l}: \mathscr{E} \rightarrow \mathscr{F} l=1,2$ are maps with $\varepsilon$-contents $N_{l}(\varepsilon), l=1,2$, then the $\varepsilon$-content $N(\varepsilon)$ of the map $\Theta=\Theta_{1}+\Theta_{2}$ is bounded by $N(\varepsilon) \leqq N_{1}(\varepsilon / 2)+N_{2}(\varepsilon / 2)$. From this estimate it follows at once that the order of $\Theta$ is less than or equal to the maximum of the orders of $\Theta_{1}$ and $\Theta_{2}$, respectively.

We conclude this section with a brief discussion of a special class of mappings from a normed space $\mathscr{E}$ into a Hilbert space $\mathscr{H}$. These mappings are of the form $(0<\lambda \leqq 1)$ 


$$
\Theta_{\lambda}(E)=T^{\lambda} \cdot \Theta(E), \quad E \in \mathscr{E},
$$

where $\Theta: \mathscr{E} \rightarrow \mathscr{H}$ is some bounded mapping, and $T$ is some positive selfadjoint operator which contains the set of vectors $\Theta(\mathscr{E}) \subset \mathscr{H}$ in its domain.

Lemma 2.3. Let $\Theta_{\lambda}, 0<\lambda \leqq 1$ be the mappings defined in relation (2.4).

i) If $\Theta_{1}$ is compact, then each $\Theta_{\lambda}, 0<\lambda \leqq 1$ is compact.

ii) If $\Theta_{1}$ is of order $q$, then $\Theta_{\lambda}, 0<\lambda \leqq 1$ is of order $q / \lambda$.

Proof. Let $P(\tau), \tau \in \mathbb{R}$ be the spectral projections of $T$, and let $d \mu(\tau)=d(\Theta(E)$, $P(\tau) \Theta(E))$ be the corresponding measure induced by the vectors $\Theta(E) \in \mathscr{H}$, where $E \in \mathscr{E}$ is kept fixed for the moment. Using Hölders inequality we obtain

$$
\left\|\Theta_{\lambda}(E)\right\|^{2}=\int d \mu(\tau) \tau^{2 \lambda} \leqq\left[\int d \mu(\tau)\right]^{1-\lambda}\left[\int d \mu(\tau) \tau^{2}\right]^{\lambda}
$$

and hence

$$
\left\|\Theta_{\lambda}(E)\right\| \leqq\|\Theta(E)\|^{1-\lambda} \cdot\left\|\Theta_{1}(E)\right\|^{\lambda} .
$$

Denoting by $N_{\lambda}(\varepsilon)$ the $\varepsilon$-content of the map $\Theta_{\lambda}$ we see from this estimate that

$$
N_{\lambda}(\varepsilon) \leqq N_{1}\left(\varepsilon^{1 / \lambda} /[2\|\Theta\|]^{1 / \lambda-1}\right)
$$

The first statement now follows from the first part of Lemma 2.1, and the second statement is an immediate consequence of the definition of order of a mapping.

\section{Modular Structure and Energy Level Density}

In this section we compare the sizes of the two sets of vectors (1.1) and (1.2). It will be convenient to regard these sets as ranges of certain maps, mapping the local algebras into $\mathscr{H}$. The order of these maps will then be taken as a measure for the size of the respective sets.

To begin with we introduce some notation: let $\mathcal{O}_{1}$ be any region in Minkowski space and let $\mathcal{O}_{2}$ be another region which contains $\mathcal{O}_{1}$. We keep these regions fixed in the subsequent discussion, but our results depend on the shape of $\mathcal{O}_{1}$ and $\mathcal{O}_{2}$. There are two parameters entering: the first one, denoted by $\delta$ and called inner distance, is the supremum of all $\tau \geqq 0$ for which the time translated regions $\mathcal{O}_{1}+t \cdot n,|t| \leqq \tau$ are contained in $\mathcal{O}_{2}$. We assume that $\delta>0$. The second parameter, called time-like extension of $\mathrm{O}_{2}$, is denoted by $d$ and is determined as follows: let $W_{\tau}, \tau \geqq 0$ be the wedge-shaped region of Minkowski-space given by

$$
W_{\tau}=\left\{x: x_{1}+\tau \geqq\left|x_{0}\right|\right\},
$$

where $x_{0}, x_{1}$ are the time and first spatial coordinate of $x$ with respect to our fixed Lorentz frame. We then define $d$ as the infimum of all $\tau \geqq 0$ for which $\mathcal{O}_{2} \subset W_{\tau} \cap$ $\left(-W_{\tau}\right)$. We assume that $d<\infty$. Note that in more than two dimensions $\mathcal{O}_{2}$ may still be unbounded.

Since the vacuum vector $\Omega$ is cyclic for the algebras $\mathscr{A}\left(\mathrm{O}_{2}\right)$ and $\mathscr{A}\left(\mathrm{O}_{2}\right)^{\prime}$ we can define an antilinear involution $S$,

$$
S A \Omega=A^{*} \Omega, \quad A \in \mathscr{A}\left(\mathcal{O}_{2}\right)
$$


familiar from the Tomita-Takesaki theory. The closure of $S$ (which we denote by the same symbol) has the polar decomposition $S=J \cdot \Delta^{1 / 2}$. The antiunitary operator $J$ is called modular conjugation, and the positive selfadjoint operator $\Delta$ modular operator. We will make use of the basic facts (cf. for example [3]) that $J \Delta^{\lambda}=\Delta^{-\lambda} J$ for $\lambda \geqq 0$, and that there holds the equality

$$
J \mathscr{A}\left(\mathcal{O}_{2}\right)^{\prime \prime} J=\mathscr{A}\left(\mathcal{O}_{2}\right)^{\prime}
$$

With the help of $\Delta$ we define as in [6] a family of mappings $\Xi_{\lambda}: \mathscr{A}\left(\mathcal{O}_{1}\right) \rightarrow \mathscr{H}$, $0<\lambda<1 / 2$

$$
\Xi_{\lambda}(A)=\Delta^{\lambda} A \Omega, \quad A \in \mathscr{A}\left(\mathcal{O}_{1}\right)
$$

For the natural value $\lambda=1 / 4$ we set $\Xi^{\natural}=\Xi_{1 / 4}$. We also consider the mapping $\Xi_{*}: \mathscr{A}\left(\mathcal{O}_{1}\right) \rightarrow \mathscr{A}\left(\mathcal{O}_{2}\right)_{*}^{\prime}$ (where $\mathscr{A}\left(\mathcal{O}_{2}\right)_{*}^{\prime}$ denotes the predual of the von Neumann algebra $\left.\mathscr{A}\left(\mathrm{O}_{2}\right)^{\prime}\right)$ given by

$$
\Xi_{*}(A)=(\Omega, A \Omega), \quad A \in \mathscr{A}\left(\mathcal{O}_{1}\right)
$$

Finally, there appears in our investigation the map $\Xi: \mathscr{A}\left(\mathcal{O}_{1}\right) \rightarrow \mathscr{H}$,

$$
\Xi(A)=\left(1+\Delta^{-1 / 2}\right)^{-1} \cdot A \Omega, \quad A \in \mathscr{A}\left(\mathcal{O}_{1}\right) .
$$

Our first result shows that for the sake of estimating the order of these mappings it is sufficient to analyze either one of them. We choose $\Xi^{\natural}$ as our standard map.

Lemma 3.1. Let $\Xi, \Xi_{*}$, and $\Xi_{\lambda}, 0<\lambda<1 / 2$ be the mappings defined in (3.4).

i) If one of these maps is compact, then all other maps are compact.

ii) Let $q, q_{*}$, and $q_{\lambda}$ be the respective orders of these maps (in an obvious notation). Setting $q^{\natural}=q_{1 / 4}$ there hold the inequalities

$$
q^{\natural} / 2 \leqq q_{*} \leqq q \leqq q_{\lambda} \leqq \max \left(\frac{1}{2 \lambda}, \frac{1}{1-2 \lambda}\right) \cdot q^{\natural} / 2 .
$$

In particular, all maps are of finite order (respectively of order 0) if anyone of them has this property.

Proof. We prove the second part of this statement, the first part can be established along the same lines. Let $A \in \mathscr{A}\left(\mathcal{O}_{1}\right)$, then there holds the equality $\left\|\Delta^{1 / 4} A \Omega\right\|^{2}=$ $(\Omega, J A J \cdot A \Omega)$. Since $J A J \in \mathscr{A}\left(\mathcal{O}_{2}\right)^{\prime}$ it follows that $\left\|\Xi^{\natural}(A)\right\|^{2} \leqq\|A\| \cdot\left\|\Xi_{*}(A)\right\|$. Denoting by $N^{\natural}(\varepsilon), N_{*}(\varepsilon)$ the $\varepsilon$-contents of the maps $\Xi^{\natural}$ and $\Xi_{*}$, respectively, we thus see that $N^{\natural}(\varepsilon) \leqq N_{*}\left(\varepsilon^{2} / 2\right)$, hence $q^{\natural} \leqq 2 q^{*}$. In the subsequent steps we proceed in a similar manner: let $A \in \mathscr{A}\left(\mathcal{O}_{1}\right)$ and $B^{\prime} \in \mathscr{A}\left(\mathcal{O}_{2}\right)^{\prime}$. Then

$$
\begin{aligned}
\left(\Omega, B^{\prime} A \Omega\right) & =\left(\left(1+\Delta^{-1 / 2}\right) B^{*} \Omega,\left(1+\Delta^{-1 / 2}\right)^{-1} A \Omega\right) \\
& =\left(\left(B^{*}+J B^{\prime}\right) \Omega,\left(1+\Delta^{-1 / 2}\right)^{-1} A \Omega\right),
\end{aligned}
$$

and consequently $\left\|\Xi_{*}(A)\right\| \leqq 2 \cdot\|\Xi(A)\|$. This shows that $q_{*} \leqq q$. Next, for $A \in \mathscr{A}\left(\mathcal{O}_{1}\right)$, we have

$$
\left(1+\Delta^{-1 / 2}\right)^{-1} A \Omega=\left(\Delta^{\lambda}+\Delta^{\lambda-1 / 2}\right)^{-1} \cdot \Delta^{\lambda} A \Omega .
$$

Since the operator $\left(\Delta^{\lambda}+\Delta^{\lambda-1 / 2}\right)^{-1}$ is bounded, we conclude that $q \leqq q_{\lambda}$. Finally, 
if $0<\lambda \leqq 1 / 4$, we write $\Delta^{\lambda} A \Omega=\Delta^{4 \lambda \cdot 1 / 4} A \Omega$, and it then follows from Lemma 2.3 that $q_{\lambda} \leqq q^{\natural} / 4 \lambda$. The case $1 / 4 \leqq \lambda<1 / 2$ can be reduced to the preceding one by making use of the equality $\Delta^{\lambda} A \Omega=J \Delta^{1 / 2-\lambda} A^{*} \Omega$. This completes the proof of the statement.

We will show that the order of these mappings, in particular the question of whether it is finite, is governed by spectral properties of the Hamiltonian. To this end we consider the family of mappings $\Theta_{\beta}: \mathscr{A}\left(\mathcal{O}_{1}\right) \rightarrow \mathscr{H}, \beta>0$ given by

$$
\Theta_{\beta}(A)=e^{-\beta H} A \Omega, \quad A \in \mathscr{A}\left(\mathcal{O}_{1}\right) .
$$

Making use of Lemma 2.3 and the fact that $e^{-\beta H}$ is bounded, we see that all maps $\Theta_{\beta}$ are compact (respectively of finite order) if anyone of them has this property. More specifically, if $q_{\beta}$ denotes the order of $\Theta_{\beta}$ we have that $q_{\beta} \geqq q_{\beta^{\prime}}$ and $\beta \cdot q_{\beta} \leqq \beta^{\prime} \cdot q_{\beta^{\prime}}$ if $\beta \leqq \beta^{\prime}$.

Let us analyze now the consequences of the assumption that the maps $\Theta_{\beta}$ are compact or of finite order, respectively. For this purpose we need two technical lemmas.

Lemma 3.2. Let $f: \mathbb{R} \rightarrow \mathbb{R}$ be any function of the form

$$
f(\omega)=\frac{1}{2 \pi} \int_{0}^{\pi} d \varphi g\left(\frac{\omega}{\cos \varphi}\right),
$$

where $g$ is the Fourier transform of an arbitrary real continuous function which has support in the interval $[-\delta, \delta]$, and let $g(0)=1$. Then there holds for $A \in \mathscr{A}\left(\mathcal{O}_{1}\right)$ the equality

$$
R \cdot A \Omega=R \cdot f(H) A \Omega+J R \cdot f(H) A^{*} \Omega,
$$

where

$$
R=\left(1+\Delta^{-1 / 2}\right)^{-1} \text {. }
$$

Remark. It follows from this result and the arguments in [5, Sect. 2] that the map $\Xi$ is nuclear if the theory satisfies the nuclearity condition in [9]. If in addition $J$ commutes with the Hamiltonian (which is the case if $\mathrm{O}_{2}$ is a wedge-shaped region of the form (3.1) [2]) one can also show that $\Xi^{\natural}$ is nuclear.

Proof. Let $\Phi$ be any vector in the domain of $S^{*}$. Then there hold for $A \in \mathscr{A}\left(\mathcal{O}_{1}\right)$ and $|t| \leqq \delta$ the equalities (since $\mathscr{A}\left(\mathcal{O}_{1}+t \cdot n\right) \subseteq \mathscr{A}\left(\mathcal{O}_{2}\right)$ )

$$
\begin{aligned}
(\Phi, U(t) S A \Omega) & =\left(\Phi, \alpha_{t}\left(A^{*}\right) \Omega\right)=\left(\Phi, S \alpha_{t}(A) \Omega\right) \\
& =\left(\alpha_{t}(A) \Omega, S^{*} \Phi\right)=\left(A \Omega, U(t)^{-1} S^{*} \Phi\right) .
\end{aligned}
$$

Moreover, since $H$ is a positive operator, the function $t \rightarrow h_{+}(t)=(\Phi, U(t) S A \Omega)$ can be analytically continued into the upper complex half-plane, and similarly the function $t \rightarrow h_{-}(t)=\left(A \Omega, U(t)^{-1} S^{*} \Phi\right)$ can be continued into the lower half plane. Thus we see that the two functions $h_{ \pm}$are the boundary values on the reals of a single function $h$ which is analytic in the cut plane $\mathbb{C} \backslash\{z: \operatorname{Im} z=0,|\operatorname{Re} z| \geqq \delta\}$.

Since the operators $U(t)$ are unitary, the functions $h_{ \pm}$are bounded, hence we 
can apply the arguments in [5, Lemma 2.3] leading to the identity for arbitrary $\tau \in[-\delta, \delta]$

$$
h_{ \pm}(0)=\frac{1}{2 \pi} \int_{0}^{\pi} d \varphi\left(h_{+}\left(\frac{\tau}{\cos \varphi}\right)+h_{-}\left(\frac{\tau}{\cos \varphi}\right)\right) .
$$

After multiplication of this equation with the Fourier transform of $g$ and integration with respect to $\tau$ we arrive at

$$
(\Phi, S A \Omega)=(\Phi, f(H) S A \Omega)+\left(A \Omega, f(H) S^{*} \Phi\right) .
$$

Choosing $\Phi=R \cdot \Psi, \Psi \in \mathscr{H}$ the statement then follows.

Lemma 3.3. There exists a numerical constant $c>0$ such that for any $\beta, 0 \leqq \beta \leqq \delta / c$ and any $\Phi \in \mathscr{H}$ there holds the inequality

$$
\inf _{f}\|f(H) \Phi\| \leqq k^{-1} \cdot\left\|e^{-\beta H} \Phi\right\|^{k} \cdot\|\Phi\|^{1-k}
$$

where $k=(1+c \beta / \delta)^{-1}$, and the infimum is to be taken with respect to all functions $f$ of the form given in (3.7).

Remark. We will show that $c \leqq \pi / \ln 4$. The smallest possible value for $c$ in the above statement is not known to us. We acknowledge discussions with $\mathbf{J}$. Michalićzek on this point.

Proof. We will exhibit an explicit sequence of functions $f_{n}, n \in \mathbb{N}$ of the form (3.7) which leads to the estimate given in the lemma.

To begin with we consider functions $s_{n}: \mathbb{R} \rightarrow \mathbb{R}$ given by (cf. [19])

$$
s_{n}(\omega)=\frac{\sin \pi \omega}{\pi \omega} \cdot \prod_{j=1}^{n}\left(1-\frac{\omega^{2}}{j^{2}}\right)^{-1}=\prod_{j=n+1}^{\infty}\left(1-\frac{\omega^{2}}{j^{2}}\right),
$$

where the second equality follows from the well-known factorization formula for the sine function. Using the Paley-Wiener theorem it is not difficult to see that the Fourier transform of each $s_{n}$ has support in $[-\pi, \pi]$ and is continuous; moreover, $s_{n}(0)=1$.

We need bounds on the functions $s_{n}$. From the second representation of $s_{n}$ we see that $\left|s_{n}(\omega)\right| \leqq 1$ if $|\omega| \leqq n$, and from the first representation we get by an elementary calculation that $\left|s_{n}(\omega)\right| \leqq(n / 2|\omega|)^{2 n-1}$ if $|\omega| \geqq n$. These two estimates lead to the uniform bound

$$
\left|s_{n}(\omega)\right| \leqq 1, \quad \omega \in \mathbb{R} .
$$

Moreover, we obtain for any $\omega_{1} \geqq 0$

$$
\inf _{n} \sup _{|\omega| \geqq \omega_{1}}\left|s_{n}(\omega)\right| \leqq 4^{1-\omega_{1}} .
$$

We will subsequently make use of this information.

Next we consider the scaled functions $g_{n}(\omega)=s_{n}(\delta \omega / \pi)$. In view of the support properties of the Fourier transforms of $s_{n}$ and the normalization $s_{n}(0)=1$ it follows that the functions 


$$
f_{n}(\omega)=\frac{1}{2 \pi} \int_{0}^{\pi} d \varphi g_{n}\left(\frac{\omega}{\cos \varphi}\right)
$$

are of the admissible form (3.7).

After these preparations we can now establish the desired estimate: let $\omega_{1} \geqq 0$ and let $P\left(\omega_{1}\right)$ be the spectral projection of $H$ corresponding to the spectrum in $\left[0, \omega_{1}\right]$. Then

$$
\begin{aligned}
\left\|f_{n}(H) \Phi\right\|^{2} & =\left\|f_{n}(H) P\left(\omega_{1}\right) \Phi\right\|^{2}+\left\|f_{n}(H)\left(1-P\left(\omega_{1}\right)\right) \Phi\right\|^{2} \\
& \leqq e^{2 \beta \omega_{1}} \sup _{\omega \leqq \omega_{1}}\left|f_{n}(\omega)\right|^{2} \cdot\left\|e^{-\beta H} \Phi\right\|^{2}+\sup _{\omega \geqq \omega_{1}}\left|f_{n}(\omega)\right|^{2}\|\Phi\|^{2} .
\end{aligned}
$$

Making use of the specific form of $f_{n}$ and the bounds on $s_{n}$ given above we see that for arbitrary $\omega_{1} \geqq 0$,

$$
\inf _{n}\left\|f_{n}(H) \Phi\right\|^{2} \leqq \frac{1}{4} e^{2 \beta \omega_{1}} \cdot\left\|e^{-\beta H} \Phi\right\|^{2}+4 e^{-(2 \ln 4 / \pi) \delta \omega_{1}} \cdot\|\Phi\|^{2} .
$$

If $\delta / \beta \geqq c=\pi / \ln 4$ the right-hand side of this inequality has a minimum for some $\omega_{1}>0$ (since $\left\|e^{-\beta H} \Phi\right\| \leqq\|\Phi\|$ ), and it is straight-forward to calculate its value. The upper bound for inf $\|f(H) \Phi\|$ obtained this way is as given in the lemma.

Combining the preceding two lemmas we can establish the desired connection between the mappings $\Theta_{\beta}, \beta>0$ and $\Xi^{\natural}$.

\section{Proposition 3.4.}

i) Let $\Theta_{\beta}, \beta>0$ be compact. Then $\Xi^{\natural}$ is compact.

ii) Let $\Theta_{\beta}, \beta>0$ be of finite $q_{\beta}$. Then $\Xi^{\natural}$ is of order $q^{\natural} \leqq 4 q_{\delta / c}$, where $\delta$ is the inner distance between $\mathcal{O}_{1}$ and $\mathcal{O}_{2}$ and $c$ is the constant in Lemma 3.3.

Remark. We recall at this point Lemma 3.1 from which an analogous result follows for all maps defined in (3.4).

Proof. To begin with we represent the map $\Xi$ as the sum of two real linear maps $\Xi_{ \pm}: \mathscr{A}\left(\mathcal{O}_{1}\right) \rightarrow \mathscr{H}$

$$
\Xi_{ \pm}(A)=\frac{1}{2} R \cdot\left(A \pm A^{*}\right) \Omega,
$$

where $R=\left(1+\Delta^{-1 / 2}\right)^{-1}$. From Lemma 3.2 we get

$$
\Xi_{ \pm}(A)=\frac{1}{2}(1 \pm J) R \cdot f(H)\left(A \pm A^{*}\right) \Omega,
$$

and consequently

$$
\left\|\Xi_{ \pm}(A)\right\| \leqq \inf \left\|f(H)\left(A \pm A^{*}\right) \Omega\right\|,
$$

where the infimum is to be taken with respect to all functions $f$ of the form (3.7). Applying to the right-hand side of this inequality Lemma 3.3. we see that for $\beta \leqq \delta / c$ and $\|A\| \leqq 1$,

$$
\left\|\Xi_{ \pm}(A)\right\| \leqq 2 \cdot k^{-1} \cdot\left\|e^{-\beta H} \frac{1}{2}\left(A \pm A^{*}\right) \Omega\right\|^{k},
$$

where $k=(1+c \beta / \delta)^{-1}$. Now let $q_{\beta}<\infty$. Then we conclude that the order $q_{ \pm}$of 
$\Xi_{ \pm}$satisfies

$$
q_{ \pm} \leqq \inf _{0 \leqq \beta \leqq \delta / c}(1+c \beta / \delta) \cdot q_{\beta} \leqq 2 q_{\delta / c} .
$$

The same bound holds for the order of $\Xi=\Xi_{+}+\Xi_{-}$because of Lemma 2.2. The second part of the statement then follows from Lemma 3.1, and by the same method one can prove the assertions about compactness.

Having analyzed the implications of spectral properties of the Hamiltonian for the modular operators we want to proceed now in the opposite direction and study how spectral properties of the modular operators lead to restrictions on the Hamiltonian. At this point we have to make use of the explicit form of the modular operators associated with wedge-shaped regions of Minkowski space. This form was found by Bisognano and Wichmann under very general assumptions [2].

Let $W_{0}$ be the wedge-shaped region defined in (3.1) for $\tau=0$. This region is invariant under the group of velocity-transformations (Lorentz-boosts) in the $x_{0}-x_{1}$ plane. We denote these transformations by $\Lambda(u), u \in \mathbb{R}$ and define their action on the space-time point $x$ by

$$
\Lambda(u) \cdot\left(\begin{array}{l}
x_{0} \\
x_{1}
\end{array}\right)=\left(\begin{array}{l}
\operatorname{ch}(2 \pi u) \cdot x_{0}+\operatorname{sh}(2 \pi u) \cdot x_{1} \\
\operatorname{sh}(2 \pi u) \cdot x_{0}+\operatorname{ch}(2 \pi u) \cdot x_{1}
\end{array}\right),
$$

all other components of $x$ remain unaffected by this transformation. Denoting by $U(\Lambda, a)$ the representation of the Poincare group on $\mathscr{H}$, we introduce the notation

$$
V(u)=U(\Lambda(u), 0), \quad U(a)=U(1, a),
$$

where $a$ is an arbitrary space-time translation. These operators satisfy the equation

$$
V(u) U(a)=U(a(u)) V(u),
$$

where we have put $a(u)=\Lambda(u) \cdot a$.

We anticipate in the following the results of Bisognano and Wichmann, quoted above, according to which the group $u \rightarrow V(u)$ is the modular group corresponding to the couple $\mathscr{A}\left(W_{0}\right), \Omega$. In other words, the operator $V(i)$ is the modular operator associated with $\mathscr{A}\left(W_{0}\right), \Omega$. This input provides the link, as we shall see, between the modular operator $\Delta$ and the Hamiltonian.

Because of the Poincare covariance of the theory it follows that the modular group corresponding to the transformed couple $\mathscr{A}\left(\Lambda \cdot W_{0}+a\right), \Omega$ is given by $u \rightarrow U(\Lambda, a) V(u) U(\Lambda, a)^{-1}$. We are in particular interested in the modular groups $V_{ \pm}(u)$ corresponding to $\mathscr{A}\left( \pm W_{\tau}\right), \Omega$ for arbitrary $\tau>0$. These groups are given by

$$
\begin{aligned}
& V_{+}(u)=U(-\underline{\tau}) V(u) U(-\underline{\tau})^{-1}=V(u) U(\underline{\tau}-\underline{\tau}(-u)), \\
& V_{-}(u)=U(\underline{\tau}) V(-u) U(\underline{\tau})^{-1}=V(-u) U(\underline{\tau}(u)-\underline{\tau}) .
\end{aligned}
$$

Here $\underline{\tau}$ denotes the spacelike vector whose components are 0 apart from $\underline{\tau}_{1}=\tau$.

Now let $B \in \mathscr{A}\left(W_{\tau}\right)$. Then it follows from the modular theory that the function $z \rightarrow V_{+}(z) B \Omega$ is analytic in the strip $0<\operatorname{Im} z<\frac{1}{2}$ and continuous at the boundaries. Next, since $z \rightarrow \underline{\tau}-\underline{\tau}(-z)$ is analytic and $\operatorname{Im}(\underline{\tau}-\underline{\tau}(z))$ is a positive time like vector if $0<\operatorname{Im} z<\frac{1}{2}$, it is an immediate consequence of the spectrum condition that the function $z \rightarrow U(\underline{\tau}-\underline{\tau}(z)) B \Omega$ is likewise analytic for $0<\operatorname{Im} z<\frac{1}{2}$ and continuous at 
the boundaries of this strip. Finally, if $P$ denotes the spectral projection of the generator of $u \rightarrow V(u)$ corresponding to any bounded subset of the spectrum we have that $z \rightarrow P \cdot V(z)$ is entire analytic. Hence we obtain from the first equation in (3.11) by analytic continuation

$$
P \cdot V_{+}(z) B \Omega=P \cdot V(z) U(\underline{\tau}-\underline{\tau}(-z)) B \Omega
$$

for $0 \leqq \operatorname{Im} z \leqq \frac{1}{2}$. Since $B \Omega$ is in the domain of $V_{+}(z)$ for the given range of $z$ we may proceed in (3.12) to the limit $P \nearrow 1$. This proves in particular that the vectors $U(\underline{\tau}-\underline{\tau}(-z)) B \Omega$ are in the domain of $V(z)$. An analogous discussion applied to $B \in \mathscr{A}\left(-W_{\tau}\right)$ leads to a relation similar to (3.12), where on the left-hand side $V_{+}$ has to be replaced by $V_{-}$, and on the right-hand side $\underline{\tau}$ by $-\underline{\tau}$, and $z$ by $-z$ (with the same restrictions on $z$ as before). We thus obtain for any $B \in \mathscr{A}\left(W_{\tau}\right) \cap \mathscr{A}\left(-W_{\tau}\right)$ and any $z, 0 \leqq \operatorname{Im} z \leqq \frac{1}{2}$ the two equations

$$
V_{ \pm}(z) B \Omega=V( \pm z) U( \pm[\underline{\tau}-\underline{\tau}(\mp z)]) B \Omega .
$$

Specializing to $z=i v, 0 \leqq v \leqq \frac{1}{2}$, we see that

$$
e^{-\sin 2 \pi v \cdot \tau H} \cdot U( \pm[1-\cos 2 \pi v] \underline{\tau}) B \Omega=V(\mp i v) V_{ \pm}(i v) B \Omega,
$$

and making use of the fact that these equations hold for any $B \in \mathscr{A}\left(W_{\tau}\right) \cap \mathscr{A}\left(-W_{\tau}\right)$ we can proceed to

$$
e^{-\sin 2 \pi v \cdot \tau H} A \Omega=V(\mp i v) V_{ \pm}(i v) U(\mp[1-\cos 2 \pi v] \underline{\tau}) A \Omega .
$$

This equation holds for $0 \leqq v<\frac{1}{4}$ and all operators

$$
A \in \mathscr{A}\left(W_{\tau}+[1-\cos 2 \pi v] \underline{\tau}\right) \cap \mathscr{A}\left(-W_{\tau}-[1-\cos 2 \pi v] \underline{\tau}\right) .
$$

(The more stringent restriction on $v$ arises from the fact that the intersection of the algebras in (3.16) becomes trivial if $\frac{1}{4} \leqq v \leqq \frac{1}{2}$ ). Denoting by $P^{+}, P^{-}$the spectral projections corresponding to the non-negative and negative spectrum of the generator of $u \rightarrow V(u)$, respectively, the information contained in (3.15) can finally be combined into the equation

$$
\begin{aligned}
e^{-\sin 2 \pi v \cdot \tau H} A \Omega= & P^{-} V(-i v) U(-\underline{\tau}) V(i v) U(\cos 2 \pi v \cdot \underline{\tau}) A \Omega \\
& +P^{+} V(i v) U(\underline{\tau}) V(-i v) U(-\cos 2 \pi v \cdot \underline{\tau}) A \Omega
\end{aligned}
$$

with the above mentioned restrictions on $A$ and $v$.

In order to get on we need the following

Lemma 3.5. Let $\mathcal{O}_{a}, \mathcal{O}_{b}$ be open regions of Minkowski space such that $\mathcal{O}_{a} \subset \mathcal{O}_{b}$ and $\mathcal{O}_{b}^{\prime}$ has a non-trivial interior, and let $\Delta_{a}, \Delta_{b}$ be the modular operators corresponding to $\mathscr{A}\left(\mathcal{O}_{a}\right), \Omega$ and $\mathscr{A}\left(\mathcal{O}_{b}\right), \Omega$, respectively. Then $\Delta_{b}^{\lambda} \Delta_{a}^{-\lambda}$ is a densely defined, bounded operator for any $0 \leqq \lambda \leqq \frac{1}{2}$, and $\left\|\Delta_{b}^{\lambda} \Delta_{a}^{-\lambda}\right\| \leqq 1$.

This result is a consequence of the fact that $\mathscr{A}\left(\mathcal{O}_{a}\right) \subset \mathscr{A}\left(\mathcal{O}_{b}\right)$. It can be verified by a standard interpolation argument, cp. [6, Lemma 2.4]. Combining this information with relation (3.17) we arrive at the crucial

Lemma 3.6.

$$
\left\|e^{-\tan 2 \pi \lambda \cdot d \cdot H} \cdot \Delta^{-\lambda}\right\| \leqq 2 \text { for } 0<\lambda<1 / 4,
$$

where $d$ denotes the time-like extension of $\mathrm{O}_{2}$. 
Remark. Since the logarithm is an operator-monotone function it follows from this result that

$$
K \leqq \frac{\tan 2 \pi \lambda}{\lambda} \cdot d \cdot H+\frac{1}{\lambda} \ln 2, \quad 0<\lambda<1 / 4
$$

where $K=-\ln \Delta$ is the generator of the modular group. This bound provides some evidence to the effect that the appropriately rescaled generators $K$ converge to the Hamiltonian $\mathrm{H}$ in the limit of large $\mathrm{O}_{2}$.

Proof. Choosing in equation (3.17) $v=\lambda, \tau=(\cos 2 \pi \lambda)^{-1} \cdot d$ we see that for each

$$
\begin{gathered}
A \in \mathscr{A}\left(\mathcal{O}_{2}\right) \subset \mathscr{A}\left(W_{d}\right) \cap \mathscr{A}\left(-W_{d}\right), \\
e^{-\tan 2 \pi \lambda \cdot d \cdot H} A \Omega=P^{-} V(-i \lambda) U(\underline{d}-\underline{\tau}) \Delta_{+}^{\lambda} A \Omega+P^{+} V(i \lambda) U(\underline{\tau}-\underline{d}) \Delta_{-}^{\lambda} A \Omega,
\end{gathered}
$$

where $\Delta_{ \pm}$are the modular operators corresponding to $\mathscr{A}\left( \pm W_{d}\right), \Omega$. Since the norm of $P^{ \pm} V( \pm i \lambda) U( \pm(\underline{\tau}-\underline{d}))$ is bounded by 1 and since $\left\|\Delta_{ \pm}^{\lambda} \Delta^{-\lambda}\right\| \leqq 1$ according to Lemma 3.5 , we conclude that

$$
e^{-\tan 2 \pi \lambda \cdot d \cdot H} A \Omega=Q \cdot \Delta^{\lambda} A \Omega,
$$

where $Q$ is a bounded operator with $\|Q\| \leqq 2$. The statement then follows from the fact that the vectors $A \Omega, A \in \mathscr{A}\left(\mathcal{O}_{2}\right)$ form a core for $\Delta^{\lambda}$.

The desired relation between the maps $\Xi^{\natural}$ and $\Theta_{\beta}, \beta>0$ can now easily be established.

\section{Proposition 3.7.}

i) Let $\Xi^{\natural}$ be compact. Then the maps $\Theta_{\beta}, \beta>0$ are compact.

ii) Let $\Xi^{\natural}$ be of finite order $q^{\natural}$. Then the maps $\Theta_{\beta}, \beta>0$ are of order $q_{\beta} \leqq q^{\natural}$. $\pi / 2 \operatorname{Arctan}(\beta / d)$, where $d$ is the time-like extension of $\mathcal{O}_{2}$.

Proof. Let $A \in \mathscr{A}\left(\mathcal{O}_{1}\right)$ and $0<\lambda<1 / 4$. Then there holds according to the preceding lemma the estimate

$$
\left\|e^{-\tan 2 \pi \lambda \cdot d \cdot H} A \Omega\right\| \leqq 2\left\|\Delta^{\lambda} A \Omega\right\|=2\left\|\Xi_{\lambda}(A)\right\| .
$$

Setting $\lambda=(2 \pi)^{-1}$ Arctan $(\beta / d)$ and making use of Lemma 3.1 the statement follows.

We conclude this section with two remarks. First we notice that if the premises of the preceding statement are satisfied for a given region $\mathcal{O}_{1}$ and some region $\mathcal{O}_{2} \supset \mathcal{O}_{1}$ with finite time-like extension, then it follows from Proposition 3.4 that these premises are satisfied for any region $\mathcal{O}_{2} \supset \mathcal{O}_{1}$, provided the inner distance between $\mathcal{O}_{1}$ and $\mathcal{O}_{2}$ is positive. This means that one may assume without loss of generality that the regions $\mathcal{O}_{1}$ and $\mathcal{O}_{2}$ are bounded.

Our second remark concerns the limit values of orders: let us fix a bounded region $\mathcal{O}_{1}$ and consider the properties of the standard maps $\Xi_{\mathscr{O}_{2}}^{\natural}$ and their respective orders $q_{\mathrm{O}_{2}}^{\natural}$ in the limit of large (bounded) regions $\mathcal{O}_{2}$. Assuming that $q_{\mathbb{O}_{2}}^{\natural}<\infty$, it follows from Lemma 3.5 that $q_{\mathscr{O}_{3}}^{\natural} \leqq q_{\mathcal{O}_{2}}^{\natural}$ if $\mathcal{O}_{3} \supset \mathcal{O}_{2}$. Hence the net $\mathcal{O}_{2} \rightarrow q_{\mathcal{O}_{2}}^{\natural}$ converges to some number $q_{\infty}^{\natural}$ if $\mathcal{O}_{2}$ tends to the whole Minkowski 
space. Similarly, the order $q_{\beta}$ of $\Theta_{\beta}, \beta>0$ converges to some number $q_{\infty}$ if $\beta$ tends to $\infty$. These asymptotic yalues are, according to Propositions 3.4 and 3.7, related by

$$
q_{\infty} \leqq q_{\infty}^{\natural} \leqq 4 q_{\infty} .
$$

On the basis of the heuristic discussion in Sect. 5 it seems plausible that $q_{\infty}=0$ if the maps $\Theta_{\beta}, \beta>0$ are of finite order. But we have not been able to verify this conjecture.

\section{Split Property and Energy Level Density}

It is worthwhile to reconsider the connection between the energy level density and the split property, disclosed in [9] and [5], in the light of the results of the preceding section. We recall that an inclusion of local algebras $\mathscr{A}\left(\mathcal{O}_{1}\right) \subset \mathscr{A}\left(\mathcal{O}_{2}\right)$ is said to be split if there exists some factor $\mathscr{M}$ of type $I$ such that $\mathscr{A}\left(\mathcal{O}_{1}\right)^{\prime \prime} \subset \mathscr{M} \subset \mathscr{A}\left(\mathcal{O}_{2}\right)^{\prime \prime}$. We will, on the one hand, establish the existence of such factors $\mathscr{M}$ under conditions on the energy level density which cover a larger class of theories than those considered in [9] and [5]. On the other hand we will show that in theories having the split property the energy level density must comply with the Haag-Swieca compactness condition [17].

Proposition 4.1. Let $\mathcal{O}$ be any bounded region of Minkowski space and let $\Theta_{\beta}, \beta>0$ be the corresponding maps defined in (3.6). If the order $q_{\beta}$ of these maps is sufficiently small for large $\beta$, then the inclusion $\mathscr{A}\left(\mathcal{O}_{1}\right) \subset \mathscr{A}\left(\mathcal{O}_{2}\right)$ is split for any region $\mathcal{O}_{1}$ whose closure is contained in the interior of $\mathcal{O}$ and any sufficiently large region $\mathrm{O}_{2}$. More specifically: the inclusion is split if the inner distance $\delta$ between $\mathcal{O}_{1}$ and $\mathcal{O}_{2}$ is such that $q_{\delta / c}<1 / 12$, where $c$ is the constant in Lemma 3.3.

Proof. Let $q_{\delta / c}<1 / 12$. It then follows from Proposition 3.4 that $q^{\natural}<1 / 3$, where $q^{\natural}$ is the order of the standard map $\Xi^{\natural}$ associated with the regions $\mathcal{O}_{1}$ and $\mathcal{O}_{2}$. From Lemma 3.1 we see that consequently $q_{*}<1 / 3$, where $q_{*}$ is the order of $\Xi_{*}$, and applying Lemma 2.1 we conclude that $\Xi_{*}$ is of type $l^{1}$, hence nuclear. But the latter fact means (cp. [6, Remark 1]) that the functional $\omega$ on $\mathscr{A}\left(\mathcal{O}_{1}\right) \otimes \mathscr{A}\left(\mathcal{O}_{2}\right)^{\prime}$ given by

$$
\omega\left(A \otimes B^{\prime}\right)=\left(\Omega, A B^{\prime} \Omega\right) \quad A \in \mathscr{A}\left(\mathcal{O}_{1}\right), B^{\prime} \in \mathscr{A}\left(\mathcal{O}_{2}\right)^{\prime}
$$

extends to a normal state on the $W^{*}$-tensor product $\mathscr{A}\left(\mathcal{O}_{1}\right)^{\prime \prime} \bar{\otimes} \mathscr{A}\left(\mathcal{O}_{2}\right)^{\prime}$. If one applies now the arguments in [5, p. 129/130] the statement follows.

Let us briefly discuss the significance of this result: if in a quantum field theory the maps $\Theta_{\beta}, \beta>0$ are of order 0 for all bounded regions $\mathcal{O}_{1}$, then it follows that the inclusions $\mathscr{A}\left(\mathcal{O}_{1}\right) \subset \mathscr{A}\left(\mathcal{O}_{2}\right)$ are split, provided the closure of $\mathcal{O}_{1}$ is contained in the interior of $\mathcal{O}_{2}$. In that case the theory is said to have the split property. It is essentially this class of theories which was studied in [9] and [5].

The present result also covers theories in which the orders of the maps $\Theta_{\beta}, \beta>0$ are different from 0 , but get small if $\dot{\beta}$ becomes large. As will be clear from the discussion in the subsequent section, this ought to be the case in theories with a maximal temperature. In that situation we still find that for each bounded region $\mathcal{O}_{1}$ there exists some bounded region $\mathcal{O}_{2}$ such that the inclusion $\mathscr{A}\left(\mathcal{O}_{1}\right) \subset \mathscr{A}\left(\mathcal{O}_{2}\right)$ is 
split. Since the inner distance $\delta$ between the regions $\mathcal{O}_{1}$ and $\mathcal{O}_{2}$ must be sufficiently large one says in this case that the theory has the distal split property. Examples of such theories have been studied in [11]. We note that the results of the heuristic discussion in the subsequent section (in particular the bounds (5.5)) seem to imply that the minimal splitting distance is proportional to the maximal temperature in the theory.

Having seen, how spectral properties of the Hamiltonian give rise to specific algebraic properties of the underlying net of local algebras, we want to discuss now how this algebraic structure leads in turn to restrictions on the Hamiltonian.

Proposition 4.2. If a quantum field theory has the (distal) split property, then the maps $\Theta_{\beta}, \beta>0$ defined in (3.6) are compact for all bounded regions $\mathcal{O}_{1}$.

Proof. The statement is based on the observation that whenever a theory has the (distal) split property, then the maps $\Xi^{\natural}$ defined in (3.4) are compact for any bounded region $\mathcal{O}_{1}$ and any (sufficiently large but bounded) region $\mathcal{O}_{2} \supset \mathcal{O}_{1}$. This fact has been established in [6 Theorem 3.3]. For the convenience of the reader we give here an elementary proof: assuming that there exists some factor $\mathscr{M}$ of type $I$ such that $\mathscr{A}\left(\mathcal{O}_{1}\right) \subseteq \mathscr{M} \subseteq \mathscr{A}\left(\mathcal{O}_{2}\right)$ we can represent the functional $\omega$ defined in relation (4.1) according to

$$
\omega\left(A \otimes B^{\prime}\right)=\sum_{i} \varphi_{i}(A) \psi_{i}\left(B^{\prime}\right),
$$

where $\varphi_{i}, \psi_{i}$ are normal states on $\mathscr{A}\left(\mathcal{O}_{1}\right)^{\prime \prime}$ and $\mathscr{A}\left(\mathcal{O}_{2}\right)^{\prime}$, respectively, and the sum on the right-hand side converges uniformly on $\mathscr{A}\left(\mathcal{O}_{1}\right)^{\prime \prime} \otimes \mathscr{A}\left(\mathcal{O}_{2}\right)^{\prime}$. Hence we see that for $A \in \mathscr{A}\left(\mathcal{O}_{1}\right)$

$$
\begin{aligned}
\left\|\Delta^{1 / 4} A \Omega\right\|^{2} & =(\Omega, J A J \cdot A \Omega) \\
& \leqq \sup \left\{\left|\omega\left(A \otimes B^{\prime}\right)\right|: B^{\prime} \in \mathscr{A}\left(\mathcal{O}_{2}\right)^{\prime},\left\|B^{\prime}\right\| \leqq\|A\|\right\} \\
& \leqq\left(\sum_{i=1}^{N}\left|\varphi_{i}(A)\right| \cdot\left\|\psi_{i}\right\|+\varepsilon \cdot\|A\|\right) \cdot\|A\|
\end{aligned}
$$

for any $\varepsilon>0$ and sufficiently large $N$. Since the unit ball of $\mathscr{A}\left(\mathcal{O}_{1}\right)^{\prime \prime}$ is weakly compact we infer from this estimate that the map $\Xi^{\natural}$ has finite $\varepsilon$-content, and hence is compact. The assertion about the maps $\Theta_{\beta}, \beta>0$ then follows from the first part of Proposition 3.7.

If in a theory the maps $\Theta_{\beta}, \beta>0$ are compact for all bounded regions $\mathcal{O}_{1}$, then this theory satisfies the compactness criterion proposed by Haag and Swieca in [17]. These authors argue that their criterion ought to be satisfied in theories having a complete particle interpretation. The split property may thus be regarded as the appropriate algebraic version of this requirement.

It is of interest in this context that the split property is known to be satisfied in many interacting theories, such as the $\phi_{2}^{4}$-theory [12], the Yukawa-theory in two dimension [22], and certain models exhibiting solitons [14].

If one looks at the proof of the preceding proposition more closely one may in fact expect that the split property comprises also some quantitative information about the maps $\Theta_{\beta}, \beta>0$ : in our argument showing that the maps $\Xi^{\natural}$ are compact 
we did not make use of specific properties of the vacuum; it holds for any cyclic and separating vector $\Omega$. On the other hand it follows from the general arguments in [6] that under the given premises there exists a dense set of vectors $\widetilde{\Omega}$ for which the corresponding maps $\widetilde{\Xi}^{\natural}$ are nuclear, respectively of finite order. In view of its distinguished rôle one may expect that the vacuum vector $\Omega$ belongs to such a set. This would then provide more specific information about the maps $\Theta_{\beta}, \beta>0$.

We conclude. this section by noting that in some models the inclusions $\mathscr{A}\left(\mathcal{O}_{1}\right) \subset \mathscr{A}\left(\mathcal{O}_{2}\right)$ are split also for certain unbounded regions $\mathcal{O}_{1}, \mathcal{O}_{2}$, such as space-like cones [10]. In that case the corresponding maps $\Xi^{\natural}$ are still compact, although the maps $\Theta_{\beta}, \beta>0$ clearly fail to have this property if $\mathcal{O}_{1}$ is unbounded.

\section{Conditions of Nuclearity}

The notion of order of a mapping has proved to be a useful tool in the preceding analysis, and we want to discuss now how big the order of the various mappings studied above actually is in models of physical interest. This heuristic discussion is based on similar arguments as in [9].

Our first hypothesis concerns the nature of boundary effects: we anticipate that perturbations of $H$ (and correspondingly of $\Omega$ ) which are located in the distant spacelike complement of a bounded region $\mathcal{O}$ have only marginal effects on the size of the set of vectors $e^{-\beta H} \mathscr{A}_{1}(\mathcal{O}) \Omega$. For the sake of estimating this size it should therefore make no substantial difference if one proceeds from the original theory to the corresponding theory in a finite spatial volume $V$ which is sufficiently large compared to the extension of $\mathcal{O}$. To be more specific: let $\mathscr{H}_{V}, H_{V}$ be the Hilbert-space and the Hamiltonian, respectively, of the finite volume theory. Then there should be a similarity transformation (i.e. a bounded, invertible map) $S$ from $\mathscr{H}_{V}$ onto $\mathscr{H}$ such that

$$
e^{-\beta H} \mathscr{A}_{1}(\mathcal{O}) \Omega \subset S e^{-\beta H_{V}} \mathscr{H}_{V, 1},
$$

where $\mathscr{H}_{V, 1}$ denotes the unit ball in $\mathscr{H}_{V}$. Moreover, since boundary effects are expected to be less significant (under normal circumstances) if one proceeds to larger systems, the similarity transformation $S$ in relation (5.1) should stay bounded in the limit of large $\mathcal{O}$, provided the ratio $V / r^{s}$, where $r$ denotes the diameter of $\mathcal{O}$ and $s$ the dimension of space, is kept large.

Our second physically motivated hypothesis relates to the properties of the operator $e^{-\beta H_{V}}$. In the framework of statistical mechanics this operator is interpreted as the (unnormalized) density matrix of the Gibbs-ensemble in the volume $V$ at temperature $\beta^{-1}$. We therefore assume that in theories admitting thermodynamical equilibrium states for arbitrarily large temperatures the operators $e^{-\beta H_{V}}$ are of trace class for all $\beta>0$. In theories with a maximal temperature this condition must be relaxed by the requirement that $e^{-\beta H_{V}}$ is a trace-class operator for sufficiently large $\beta$.

In either case the trace of this operator (the partition function) is to be interpreted as the exponential function of the free energy, multiplied by $-\beta$. One therefore expects that in theories with normal thermodynamical properties there holds for 
large volumes $V$ the bound

$$
\operatorname{Tr} e^{-\beta H_{V}} \leqq e^{V \cdot \phi(\beta)},
$$

where $\phi(\beta)$ is some theory dependent function which tends to infinity as $\beta^{-1}$ approaches the maximal temperature in the given theory, respectively infinite temperatures.

This heuristic input is the same as in [9]. But the mathematical implications which can be drawn from it about the nature of the maps $\Theta_{\beta, \mathcal{O}}: \mathscr{A}(\mathcal{O}) \rightarrow \mathscr{H}$ defined in (3.6) are more specific than those stated in [9]. (Since $\mathcal{O}$ is arbitrary we label these maps in the subsequent discussion by the region corresponding to their respective domains.)

First we notice that relation (5.1) implies that the map $\Phi_{V}: \mathscr{A}(\mathcal{O}) \rightarrow \mathscr{H}_{V}$ given by

$$
\Phi_{V}(A)=e^{\beta H_{V}} S^{-1} e^{-\beta H} A \Omega, \quad A \in \mathscr{A}(\mathcal{O})
$$

is bounded by 1 . Second, the assumption that the operators $e^{-\beta H_{V}}$ are of trace class for any $\beta>0$ implies that these operators are, for fixed $\beta$, elements of all Schatten-von Neumann classes [18].

Making use of the spectral theorem and the remark following after relation (2.3) we thus see that each map $e^{-\beta H_{V}}: \mathscr{H}_{V} \rightarrow \mathscr{H}_{V}$ is of type $s$ (order 0). But the maps of a fixed type form an ideal in the space of all bounded maps between Banach spaces [20]. Hence the map $\Theta_{\beta, \mathcal{O}}$, which is obtained by composing $e^{-\beta H_{V}}$ with the bounded maps $\Phi_{V}$ and $S$, respectively, is of type $s$ (order 0 ) too. We recall in this context that if $\Theta_{\beta, \mathcal{O}}$ is of type $s$ for some value of $\beta$, then it is of type $s$ for all $\beta>0$.

On the basis of relations (5.1) and (5.2) one can also estimate the p-norms of these maps. From the definition (2.3) it follows by a straightforward calculation that for any $p>0$,

$$
\left\|\Theta_{\beta, \mathcal{O}}\right\|_{p} \leqq\|S\| \cdot\left\|\Phi_{V}\right\| \cdot\left(\operatorname{Tr} e^{-p \beta H_{V}}\right)^{1 / p} .
$$

But $\left\|\Phi_{V}\right\| \leqq 1$, and by assumption the similarity transformations $S$ stay bounded for arbitrarily large regions $\mathcal{O}$, provided $V / r^{s}$ is kept large. For large diameters $r$ of $\mathcal{O}$ we can therefore proceed from (5.4) (by fixing the ratio $V / r^{s}$ ) to the estimate

$$
\left\|\Theta_{\beta, \mathcal{O}}\right\|_{p} \leqq \text { const } e^{r \boldsymbol{s} \phi(p \cdot \beta) / p}
$$

where we have absorbed the constant $V / r^{s}$ in the function $\phi$.

We note that in generic cases (e.g. if Stefan-Boltzmann's law holds at high temperatures) the function $\phi$ should exhibit a power-like singularity at $\beta=0$. For $p=1$ the estimate (5.5) then reproduces the bounds on the nuclearity index given in [9].

The above reasoning can also be applied to theories with a maximal temperature, the only difference being that there $e^{-\beta H_{V}}$ is a trace-class operator only if $\beta>\beta_{\min }$ (the inverse of the maximal temperature). Relation (5.5) also holds in these cases if $p>\beta_{\min } / \beta$. From this we see that the order of the maps $\Theta_{\beta, \mathcal{O}}$ can still be made arbitrarily small provided $\beta$ is chosen to be sufficiently large.

Let us briefly discuss how these conclusions have to be modified if one replaces in the above reasoning the vacuum state by some equilibrium state at temperature 
$T, T \cdot \beta_{\min }<1$. We denote the corresponding cyclic vector by $\Omega_{T}$ and the generator of the time-translations annihilating $\Omega_{T}$ by $H_{T}$. In analogy to the preceding discussion we consider the local excitations of $\Omega_{T}$, i.e. the set of vectors $A \Omega_{T}, A \in \mathscr{A}_{1}(\mathcal{O})$. But now it no longer makes sense to restrict the total energy of these states (which is infinite) ${ }^{1}$. Instead we restrict the energy transferred by the operators $A$ to $\Omega_{T}$ by taking time-averages $\int d t f(t) \alpha_{t}(A)$ with a suitable testfunction $f(t)$ whose Fourier transform $\tilde{f}(\omega)$ decreases exponentially. In view of the invariance of $\Omega_{T}$ under time-translations we have that

$$
\int d t f(t) \alpha_{t}(A) \Omega_{T}=\tilde{f}\left(H_{T}\right) A \Omega_{T},
$$

and we are thus led to consider as analogue of $\Theta_{\beta, \mathcal{O}}$ the maps

$$
A \in \mathscr{A}(\mathcal{O}) \rightarrow e^{-\beta\left|H_{T}\right|} A \Omega_{T}, \beta>0,
$$

where $\left|H_{T}\right|$ denotes the modulus of $H_{T}$. A similar argument as in the case of the vacuum state leads to the conclusion that these maps ought to have finite $p$-norm, $p>2 T \cdot \beta_{\min }$, provided $\beta$ is sufficiently large, viz. $\beta>\beta_{\min } / p$. If there are no limitations on the temperature $T$, the maps (5.7) thus ought to be of order 0 for any $T \geqq 0$ and any $\beta>0$.

If the latter condition is satisfied it follows that also the maps

$$
A \in \mathscr{A}(\mathcal{O}) \rightarrow e^{-\beta H_{T}} A \Omega_{T}, \quad 0<\beta<1 / 2 T
$$

are of order 0 , despite of the fact that the operator $e^{-\beta H_{T}}$ is unbounded. For the proof of this assertion we recall that $e^{-H_{T} / T}$ is the modular operator associated with $\Omega_{T}$ and the algebra $\mathscr{A}$ of all local operators (since the state represented by $\Omega_{T}$ satisfies the KMS-condition for temperature $T$ ) [3]. Let $J$ be the associated modular conjugation and let $P^{ \pm}$be the projections onto the positive and negative spectrum of $H_{T}$, respectively. Making use of the Tomita-Takesaki theory we have that for any $A \in \mathscr{A}$ there holds the equality

$$
\begin{aligned}
J P^{+} e^{-\beta H_{T}} A^{*} \Omega_{T} & =J P^{+} e^{-\beta H_{T}} \cdot e^{H_{T} / 2 T} J A \Omega_{T} \\
& =P^{-} e^{(\beta-1 / 2 T) H_{T}} A \Omega_{T} .
\end{aligned}
$$

Now if the map (5.7) is of order 0, then the map $A \in \mathscr{A}(\mathcal{O}) \rightarrow P^{+} e^{-\beta H_{T}} A \Omega_{T}$ is also of order 0 since $P^{+} e^{-\beta H_{T}}=P^{+} e^{-\beta\left|H_{T}\right|}$. Moreover, since $J$ is bounded, the equality (5.9) implies that the map $A \in \mathscr{A}(\mathcal{O}) \rightarrow P^{-} e^{(\beta-1 / 2 T) H_{T}} A \Omega_{T}$ is, for any $\beta>0$, of order 0 too. Bearing in mind that the maps of order 0 form a linear space we thus conclude that the maps (5.8) are of order 0 for the given range of $\beta$.

It is clear that these limitations on $\beta$ cannot be relaxed. Since $e^{-H_{T} / 2 T} A \Omega_{T}=$ $J A^{*} \Omega_{T}$ the map (5.8) is not even compact if $\beta \geqq 1 / 2 T$. In theories with a maximal temperature there arise further restrictions on the admissible range of $\beta$ : one finds by the same arguments as before that the maps (5.8) have finite $p$-norm if $p>4 T \cdot \beta_{\min }$ and if $\beta$ satisfies $\beta_{\min } / p<\beta<1 / 2 T-\beta_{\min } / p$. It is note-worthy that the latter condition is always satisfied for the (according to the Araki theory of cones [1] "natural") value $\beta=1 / 4 T$.

Having seen how in the case of temperature states the number of local degrees

\footnotetext{
1 We recall that $H_{T}$ does not have the meaning of the observable "energy" [16]
} 
of freedom of a theory is encoded in properties of the maps (5.8), let us now turn back to the vacuum situation. The results of our heuristic considerations can be summarized in the following variant of the nuclearity condition in [9].

Condition of Energy Nuclearity. Let $\mathcal{O}$ be any bounded region of Minkowski space. Then the maps $\Theta_{\beta, \mathcal{O}}: \mathscr{A}(\mathcal{O}) \rightarrow \mathscr{H}$ given by

$$
\Theta_{\beta, \mathcal{O}}(A)=e^{-\beta H} A \Omega, \quad A \in \mathscr{A}(\mathcal{O})
$$

must be of arbitrarily small order if $\beta>0$ is sufficiently large.

It is clear from the above discussion that there should hold a similar condition involving the temperature states. One can also give quantitative versions of this condition in terms of the $p$-norms of $\Theta_{\beta, \mathcal{O}}, \mathrm{cp}$. relation (5.5). The above condition must therefore be regarded as a very weak requirement. It does not seem, however, to follow from the nuclearity condition stated in [9], although it has the same heuristic basics. We therefore propose this alternative formulation since, on the one hand, it comprises more specific information about the properties of the maps $\Theta_{\beta, \mathcal{O}}$. On the other hand, it seems to cover a larger class of theories of potential physical interest, including theories with a maximal temperature.

Our condition can also be stated in terms of the modular operators associated with the local algebras and the vacuum state.

Condition of Modular Nuclearity. Let $\mathcal{O}_{1} \subset \mathcal{O}_{2}$ be arbitrary bounded regions of Minkowski space and let $\Xi_{\mathcal{O}_{2}, \mathcal{O}_{1}}: \mathscr{A}\left(\mathcal{O}_{1}\right) \rightarrow \mathscr{H}$ be the maps given by

$$
\Xi_{\mathcal{O}_{2}, \mathcal{O}_{1}}(A)=\Delta_{\mathscr{O}_{2}}^{1 / 4} A \Omega, \quad A \in \mathscr{A}\left(\mathcal{O}_{1}\right)
$$

where $\Delta_{\mathcal{O}_{2}}$ is the modular operator associated with the pair $\mathscr{A}\left(\mathcal{O}_{2}\right), \Omega$. These maps must be of arbitrarily small order if the inner distance between $\mathcal{O}_{1}$ and $\mathcal{O}_{2}$ is sufficiently large.

It is clear from the results of Sect. 3 that this condition is equivalent to the preceding one involving the Hamiltonian. Those results, combined with the bounds (5.5), also suggest obvious quantitative formulations of the condition which might be of use.

What we would like to point out here is the fact that our modular nuclearity condition is local in the sense that it only requires information about the restriction of the vacuum functional $\omega_{0}$ to the local algebras: given these partial states, the relevant modular operators are fixed and the nuclearity condition can be checked. This check does not hinge on the explicit knowledge of the Hamiltonian.

The latter remark is substantiated by the following observation: if $\Omega$ complies with the condition of modular nuclearity, then the same condition is satisfied by a dense set of vectors $\widetilde{\Omega} \in \mathscr{H}$. To verify this we pick any unitary operator $V$ with bounded localization region $\mathcal{O}_{0}$ and set $\widetilde{\Omega}=V \cdot \Omega$. Given $\mathcal{O}_{1}$ we choose $\mathcal{O}_{2} \supset \mathcal{O}_{1} \cup \mathcal{O}_{0}$. The modular operator $\tilde{\Delta}_{\mathcal{O}_{2}}$ corresponding to the pair $\mathscr{A}\left(\mathcal{O}_{2}\right), \widetilde{\Omega}$ is then given by $\tilde{\Delta}_{\mathcal{O}_{2}}=V \Delta_{\mathcal{O}_{2}} V^{*}$, and consequently

$$
\tilde{\Delta}_{\mathscr{O}_{2}}^{1 / 4} A \tilde{\Omega}=V \Delta_{\mathscr{O}_{2}}^{1 / 4} \cdot V^{*} A V \Omega
$$

for all $A \in \mathscr{A}\left(\mathcal{O}_{1}\right)$. But the map $A \rightarrow V^{*} A V$ of $\mathscr{A}\left(\mathcal{O}_{1}\right)$ into $\mathscr{A}\left(\mathcal{O}_{1} \cup \mathcal{O}_{0}\right)$ is bounded 
and it thus follows from relation (5.10) that $\widetilde{\Omega}$ satisfies the condition of modular nuclearity if the vacuum vector $\Omega$ does. Since the local operators act irreducibly on $\mathscr{H}$ the set of vectors of the form of $\widetilde{\Omega}$ is dense, hence the assertion follows.

The fact that the formulation of our condition of modular nuclearity does not depend on the existence of unitary time-translations (a Hamiltonian) suggests that it also prevails in theories where the underlying space-time manifold does not admit such a global symmetry. It therefore may be of interest in the structural analysis of generally covariant quantum field theories [13]. For example, it follows directly from the condition of modular nuclearity (i.e. without relying on the Hamiltonian) that the theory has the distal split-property if the local algebras are factors [6].

\section{Appendix}

It is the purpose of this Appendix to demonstrate that the condition of energy nuclearity, as stated in Sect. 5, is satisfied in free field theory. We discuss here the theory of a scalar particle of mass $\mu \geqq 0$. The case $\mu=0$ is of particular interest since in that case the secondary rôle of boundary effects, anticipated in our heuristic discussion, is less obvious due to the presence of long-range correlations.

Our reasoning is similar to the arguments in [9] and [7], and we therefore confine ourselves to indicating the necessary modifications. We adopt the notations in [7]. Let $\mathscr{K}$ be a complex Hilbert space (the single-particle space), let $J$ be an anti-unitary involution, and let $\omega$ be some positive operator (the single particle Hamiltonian) commuting with $J$.

The symmetric Fock space over $\mathscr{K}$ is denoted by $\mathscr{H}$ and the distinguished vector representing the vacuum by $\Omega$. On $\mathscr{K}$ there act the familiar creation and annihilation operators $a^{*}(f)$ and $a(f), f \in K$, and the corresponding unitary Weyl operators

$$
W(f)=\exp \left(i\left[a^{*}(f)+a(f)\right]^{-}\right) .
$$

By second quantization of $\omega$ we get a positive selfadjoint operator $H$ (the Hamiltonian) for which we have $H \Omega=0$ and

$$
e^{i t H} W(f) e^{-i t H}=W\left(e^{i t \omega} f\right), \quad t \in \mathbb{R} .
$$

Let $\mathscr{L}_{\phi}$ and $\mathscr{L}_{\pi}$ be closed subspaces of $\mathscr{K}$ which are stable under the action of $J$. We consider the real-linear subspace

$$
\mathscr{L}=(1+J) \mathscr{L}_{\phi}+(1-J) \mathscr{L}_{\pi},
$$

and the corresponding von Neumann algebra

$$
\mathscr{W}(\mathscr{L})=\{W(f): f \in \mathscr{L}\}^{\prime \prime} .
$$

This algebra is to be interpreted as the local algebra $\mathscr{A}(\mathcal{O})$ attached to a given space-time region $\mathcal{O}$ for a specific choice of the spaces $\mathscr{L}_{\phi}$ and $\mathscr{L}_{\pi}$, see below. We define for any $\beta>0$ maps $\Theta_{\beta}: \mathscr{W}(\mathscr{L}) \rightarrow \mathscr{H}$, setting

$$
\Theta_{\beta}(A)=e^{-\beta H} A \Omega, \quad A \in \mathscr{W}(\mathscr{L}) .
$$

Denoting by $E_{\phi}, E_{\pi}$ the projections onto $\mathscr{L}_{\phi}$ and $\mathscr{L}_{\pi}$, respectively, we have in analogy to [7, Theorem 2.1]: 
Lemma. Let $\left|E_{\phi} e^{-\beta \omega}\right|^{p}$ and $\left|E_{\pi} e^{-\beta \omega}\right|^{p}$ be trace class operators for some $0<p \leqq 1$. Then the map $\Theta_{\beta}$ has finite p-norm and

$$
\left\|\Theta_{\beta}\right\|_{p} \leqq \operatorname{det}\left(1-\left|E_{\phi} e^{-\beta \omega}\right|^{p}\right)^{-2 / p} \cdot \operatorname{det}\left(1-\left|E_{\pi} e^{-\beta \omega}\right|^{p}\right)^{-2 / p} .
$$

Proof. Let $T$ be the "least upper bound" [7, p. 316] of the operators $\left|E_{\phi} e^{-\beta \omega}\right|$ and $\left|E_{\pi} e^{-\beta \omega}\right|$. As a consequence of the stated assumptions, $|T|^{p}$ is of trace class and $\|T\|<1$ (since $\omega$ is positive). Choosing in $\mathscr{K}$ as orthonormal basis the eigenvectors $e_{i}$ of $T$ corresponding to the eigenvalues $t_{i}$ (counted including multiplicity) we obtain, by taking tensor products of these single-particle vectors, an orthonormal basis $\Phi_{(n)}$ in $\mathscr{H}$. Here $(n)=\left(n_{1}, n_{2}, \ldots, n_{i}, \ldots\right)$ is a multi-index specifying the "occupation numbers" $n_{i}$ of the "modes" $e_{i}$ in the state $\Phi_{(n)}$. On account of the equality

$$
\Theta_{\beta}(A)=\sum_{(n)}\left(\Phi_{(n)}, e^{-\beta H} A \Omega\right) \cdot \Phi_{(n)}
$$

it is clear that

$$
\left\|\Theta_{\beta}(A)\right\|_{p}^{p} \leqq \sum_{(n)} \sup \left\{\left|\left(\Phi_{(n)}, e^{-\beta H} A \Omega\right)\right|^{p}: A \in \mathscr{W}(\mathscr{L})_{1}\right\}
$$

Moreover, applying the arguments in the proof of Theorem 2.1 in [7] and Lemma 1 in the Appendix of [9] it follows that

$$
\sup \left\{\left|\left(\Phi_{(n)}, e^{-\beta H} A \Omega\right)\right|: A \in \mathscr{W}(\mathscr{L})_{1}\right\} \leqq \prod_{i}\left(n_{i}+1\right) t_{i}^{n_{i}},
$$

where we adopt the convention that $0^{0}=1$. From this estimate and the fact that $p \leqq 1$ we conclude that

$$
\left\|\Theta_{\beta}\right\|_{p}^{p} \leqq \operatorname{det}\left(1-T^{p}\right)^{-2}<\infty .
$$

As in [7; p. 318] the given bound on $\|\Theta\|_{p}$ then follows from the fact that $T$ is the least upper bound of $\left|E_{\phi} e^{-\beta \omega}\right|$ and $\left|E_{\pi} e^{-\beta \omega}\right|$, respectively.

With the help of this lemma we have reduced the problem of determining the nuclearity properties of the maps $\Theta_{\beta}, \beta>0$ to a standard problem on the single particle space. This result can be applied to models of an arbitrary number of free scalar Bose-particles. We study here only the case of a single particle of mass $\mu \geqq 0$ in $s>1$ spatial dimension. In that case $\mathscr{K}$ can be identified with the space $L^{2}\left(\mathbb{R}^{s}\right)$ of single-particle wave-functions $f, J$ is the operator of complex conjugation in configuration space, i.e. $(J f)(\underline{x})=f(\underline{x})^{*}$, and the action of $\omega$ is given by

$$
(\widetilde{\omega f})(\underline{p})=\left(|\underline{p}|^{2}+\mu^{2}\right)^{1 / 2} \cdot \tilde{f}(\underline{p}) \text {, }
$$

where $\tilde{f}$ denotes the Fourier transform of $f$.

It suffices to consider the local algebras $\mathscr{A}(\mathcal{O})$ associated with double cones $\mathcal{O}$ whose bases $\underline{O}$ lie in the time zero plane. The corresponding subspaces $\mathscr{L}_{\phi}, \mathscr{L}_{\pi}$ are given by

$$
\mathscr{L}_{\phi}=\overline{\omega^{-1 / 2} \mathscr{D}(\underline{\mathcal{O}})}, \quad \mathscr{L}_{\pi}=\overline{\omega^{1 / 2} \mathscr{D}(\underline{\mathcal{O}})}
$$

where $\mathscr{D}(\underline{\mathcal{O}})$ is the space of all testfunctions with support in the bounded region $\underline{\mathcal{O}} \subset \mathbb{R}^{s}$; and the bar denotes closure. 
Lemma. Let $E_{\phi}, E_{\pi}$ be the orthogonal projections onto the spaces $\mathscr{L}_{\phi}$ and $\mathscr{L}_{\pi}$, respectively, defined in (A.7) and let $\omega$ be the positive operator defined in (A.6). Then $\left|E_{\phi} e^{-\beta \omega}\right|$ and $\left|E_{\pi} e^{-\beta \omega}\right|$ are, for any $\beta>0$, elements of all Schatten classes (i.e. the $p^{\text {th }}$ powers of these operators are of trace-class for any $p>0$ ).

Proof. Let $\chi_{0} \in \mathscr{S}\left(\mathbb{R}^{s}\right)$ be any test function which is equal to 1 on the region $\underline{Q}$ and let $\chi$ be the corresponding multiplication operator in configuration space, $(\chi f)(\underline{x})=\chi_{0}(\underline{x}) \cdot f(\underline{x})$. A straightforward calculation shows that for any $n \in \mathbb{N}$ the operators

$$
\begin{aligned}
& h_{n}=\omega^{1 / 2} \cdot\left(1+\omega^{2}\right)^{(n-1) s} \chi\left(1+\omega^{2}\right)^{-n s} \cdot \omega^{-1 / 2}, \\
& k_{n}=\omega^{-1 / 2} \cdot\left(1+\omega^{2}\right)^{(n-1) s} \chi\left(1+\omega^{2}\right)^{-n s} \omega^{1 / 2}
\end{aligned}
$$

are in the Hilbert-Schmidt class. Moreover, it follows immediately from the definition of the spaces $\mathscr{L}_{\phi}$ and $\mathscr{L}_{\pi}$ that there hold the equalities

$$
\begin{aligned}
& E_{\phi} e^{-\beta \omega}=E_{\phi} h_{1} \cdot h_{2} \cdots h_{n} \cdot\left(1+\omega^{2}\right)^{n s} e^{-\beta \omega}, \\
& E_{\pi} e^{-\beta \omega}=E_{\pi} k_{1} \cdot k_{2} \cdots k_{n} \cdot\left(1+\omega^{2}\right)^{n s} e^{-\beta \omega} .
\end{aligned}
$$

Since $\omega$ is positive, the expression involving $\omega$ which appears on the right-hand side of these equalities is a bounded operator for any $\beta>0$. Hence the operators $E_{\phi} e^{-\beta \omega}$ and $E_{\pi} e^{-\beta \omega}$ can be represented as a product of an arbitrary number of Hilbert-Schmidt operators. This proves the statement.

Combining the previous two lemmas we arrive at the

Proposition. In the quantum field theory of a free scalar particle of mass $\mu \geqq 0$ in $s>1$ space dimensions the maps $\Theta_{\beta}: \mathscr{A}(\mathcal{O}) \rightarrow \mathscr{H}$ given by

$$
\Theta_{\beta}(A)=e^{-\beta H} A \Omega, \quad A \in \mathscr{A}(\mathcal{O})
$$

are of order 0 for any $\beta>0$ and any bounded space-time region $\mathcal{O}$.

Remark. If $\mu>0$ this result holds also if $s=1$. Moreover, by a refined analysis one can give quantitative bounds on the $p$-norms of $\Theta_{\beta}, \beta>0$ which are consistent with the out-come of the heuristic discussion in Sect. 5.

Acknowledgements. The first named author (D. B.) would like to thank S. Doplicher, Università di Roma I, for shown hospitality, and the CNR of Italy for financial support. The second named author (C. D.) would like to thank the members of the II. Institut für Theoretische Physik, Universität Hamburg and K. Fredenhagen, Freie Universität Berlin, for hospitality and the A. von Humboldt Foundation for financial support.

\section{References}

1. Araki, H.: Some properties of modular conjugation operators of a von Neumann algebra and a non-commutative Radon-Nikodym derivative with a chain rule. Pac. J. Math. 50, 309-354 (1974)

2. Bisognano, J. J., Wichmann, E. H.: On the duality condition for a hermitian scalar field. J. Math. Phys. 16, 985-1007 (1975); On the duality condition for quantum fields. J. Math. Phys. 17, 303-321 (1976)

3. Bratteli, O., Robinson, D. W.: Operator algebras and quantum statistical mechanics I. Berlin, Heidelberg, New York: Springer 1979 
4. Buchholz, D.: On particles, infraparticles, and the problem of asymptotic completeness. In: VIIIth International Congress on Mathematical Physics, Marseille 1986. Singapore: World Scientific 1987

5. Buchholz, D., D'Antoni, C., Fredenhagen, K.: The universal structure of local algebras. Commun. Math. Phys. 111, 123-135 (1987)

6. Buchholz, D., D'Antoni, C., Longo, R.: Nuclear maps and modular structures, I: General properties. J. Funct. Anal. (to appear)

7. Buchholz, D., Jacobi, P.: On the nuclearity condition for massless fields. Lett. Math. Phys. 13, 313-323 (1987)

8. Buchholz, D., Junglas, P.: Local properties of equilibrium states and the particle spectrum in quantum field theory. Lett. Math. Phys. 11, 51-58 (1986)

9. Buchholz, D., Wichmann, E. H.: Causal independence and the energy-level density of states in quantum field theory. Commun. Math. Phys. 106, 321-344 (1986)

10. D'Antoni, C., Fredenhagen, K.: Charges in spacelike cones. Commun. Math. Phys. 94, 537-544 (1984). Erratum: ibid. 96, 566 (1984)

11. D'Antoni, C., Doplicher, S., Fredenhagen, K., Longo, R.: Convergence of local charges and continuity properties of $W^{*}$-inclusions. Commun. Math. Phys. 116, 325-348 (1987). Erratum: ibid. $110,175(1988)$

12. Driessler, W.: Duality and absence of locally generated superselection sectors for CCR-type algebras. Commun. Math. Phys. 70, 213-220 (1979)

13. Fredenhagen, K., Haag, R.: Generally covariant quantum field theory and scaling limits. Commun. Math. Phys. 108, 91-115 (1987)

14. Fröhlich, J.: Quantum theory of non-linear invariant wave (field) equations or: Superselection sectors in constructive quantum field theory. In: Invariant wave equations. Lecture Notes in Physics, Vol. 73. Berlin, Heidelberg, New York: Springer 1978

15. Haag, R., Kastler, D.: An algebraic approach to quantum field theory. J. Math. Phys. 5, 848-861 (1964)

16. Haag, R., Hugenholtz, N. H., Winnink, M.: On the equilibrium states in quantum statistical mechanics. Commun. Math. Phys. 5, 215-236 (1967)

17. Haag, R., Swieca, A. J.: When does a quantum field theory describe particles? Commun. Math. Phys. 1, 308-320 (1965)

18. Jarchow, H.: Locally convex spaces. Stuttgart: Teubner 1981

19. Lax, P. D.: An inequality for functions of exponential type. Commun. Pure Appl. Math. 16, 241-246 (1963)

20. Pietsch, A.: Nuclear locally convex spaces. Berlin, Heidelberg, New York: Springer 1972

21. Sewell, G. I.: Quantum fields on manifolds. Ann. Phys. 141, 201-224 (1982)

22. Summers, J. S.: Normal product states for fermions and twisted duality for CCR and CAR algebras with application to the Yukawa ${ }_{2}$ quantum field model. Commun. Math. Phys. 86, 111-141 (1982)

Communicated by $\mathrm{H}$. Araki

Received August 19, 1989 